\title{
Different mechanisms of transmission of the microsporidium Octosporea bayeri: a cocktail of solutions for the problem of parasite permanence
}

\author{
D. B. VIZOSO*, S. LASS and D. EBERT \\ Département de Biologie, Unité d'Ecologie and Evolution, Université de Fribourg, Chemin du Musée 10, \\ CH-1700 Fribourg, Switzerland
}

(Received 1 fune 2004; revised 2 September 2004; accepted 3 September 2004)

S UMMAR Y

Periods of low host density impose a constraint on parasites with direct transmission, challenging their permanence in the system. The microsporidium Octosporea bayeri faces such constraint in a metapopulation of its host, the cladoceran Daphnia magna, where ponds frequently lose their host population due to ponds drying out in summer and freezing in winter. We conducted experiments aimed to investigate the mechanisms of transmission of $O$. bayeri, and discuss how these mechanisms could contribute to the parasite's permanence in the system. Spores accumulate in the fat cells and the ovaries of the host, and vary in morphology, possibly corresponding to 3 different spore types. Horizontal transmission occurred through the release of spores from dead hosts, with the proportion of infected hosts depending on the spore dose. Further, spores are able to persist outside the host both in dry and wet conditions. Vertical transmission occurred to both parthenogenetic and sexual offspring. The former were invariably infected, while the sexually produced resting eggs (= ephippia) had a less efficient transmission. The parasite may be carried by the ephippia, and thus disperse to new ponds together with the host. Together, these mechanisms may allow the parasite to endure periods of harsh environmental conditions both outside and inside the host.

Key words: vertical and horizontal transmission, parasite permanence, spore survival, metapopulation, Octosporea bayeri, Daphnia magna.

\section{INTRODUCTION}

Successful host-to-host transmission is critical for the survival of parasites. Low host densities and host extinction can produce severe bottlenecks for parasites if transmission requires the presence of a minimum density of susceptible hosts (e.g. if transmission is density dependent, Anderson \& May, 1978, 1981, 1986; Onstad et al. 1990). In microparasites that infect new hosts via spores released to the environment, horizontal transmission depends on the rate of spore contact with new hosts (Anderson \& May, 1981; Regoes et al. 2003). At high host densities, the contact between susceptible hosts and spores might be frequent enough for parasite permanence, even if spore survival in the environment is short. At low densities, however, the contact will be reduced and parasites with spores that die before infecting a susceptible host will disappear from the population. Frequent periods of low host density may thus select for an increase in spore longevity. A similar scenario has been recently

* Corresponding author. Present address: Institut für Zoologie und Limnologie, Abteilung Ultrastrukturforschung und Evolutionsbiologie, Universität Innsbruck, Technikerstrasse 25, 6020 Innsbruck, Austria. E-mail:dita.vizoso@uibk.ac.at modelled for the Hantavirus-Vole system (Sauvage et al. 2003). Their theoretical results confirm the argument above: by adding survival outside the host, the permanence and prevalence of the pathogen in the population increased.

In microsporidia, spores released to the environment are characterized by a complex spore wall, which has been suggested as an adaptation for survival outside the host (Bigliardi \& Sacchi, 2001; Maddox, 2002). The resistance to environmental stress (often referred to as environmental persistence) has been studied in relatively few species of microsporidia. Brooks (1988) reviewed the effects of different abiotic factors on the environmental persistence of microsporidia infecting insects. According to this review, spore survival could vary from hours to years, depending on the species and the combination of abiotic factors to which the spores were subjected.

Relying solely on propagules to persist in a fluctuating environment, however, may prove risky. Environmental persistence of spores is likely to trade-off with spore production and/or within-host growth (Bonhoeffer, Lenski \& Ebert, 1996), and thus reduce parasite competitiveness (Frank, 1996). During periods of high host density more competitive parasites with less resistant spores could be favoured, as they will produce more spores. Similarly, co-infections would lead to within-host 
Table 1. Summary of experiments and results

\begin{tabular}{|c|c|c|}
\hline Number & Experiment & Main result \\
\hline 1 & Spore-dose & $\begin{array}{l}\text { Horizontal transmission depends on the density of } \\
\text { propagules present in the medium. }\end{array}$ \\
\hline 2 & $\begin{array}{l}\text { Timing of horizontal transmission } \\
\text { and the potential influence of } \\
\text { parasite isolate and host sex }\end{array}$ & $\begin{array}{l}\text { Horizontal transmission only occurs after host death. } \\
\text { Neither host sex nor parasite isolate influenced infectivity. }\end{array}$ \\
\hline 3 & Spores in aqueous solution & $\begin{array}{l}\text { Spores are viable for at least } 24 \text { days outside the host, } \\
\text { in aqueous solution. }\end{array}$ \\
\hline 4 & Desiccation of spores & Spores remain viable after at least 5 weeks of desiccation. \\
\hline 5 & $\begin{array}{l}\text { Transmission to parthenogenetic } \\
\text { offspring }\end{array}$ & Transmission likely occurs in the ovary. \\
\hline 6 & Transmission to sexual offspring & Resting, sexual, eggs are infected vertically. \\
\hline
\end{tabular}

competition, where parasites with low environmental persistence but high within-host reproduction could be favoured. Moreover, environmental persistence is limited, with a gradual decay of infectivity with time. During extended periods of low host density the parasite might thus be lost from the system.

The evolution of vertical transmission has been suggested as another adaptation to reduce the risks of parasite extinction due to low host densities (Lucarotti \& Andreadis, 1995; van Baalen, 2000; Agnew et al. 2002). Vertical transmission (from parent to offspring) may help a parasite to persist in a host population even if host density is very low. Exclusive vertical transmission, however, also bears risks. If host density increases, the harmful effect of parasites may lower the competitiveness of infected hosts relative to that of uninfected hosts, reduce parasite prevalence and eventually lead to parasite extinction. For that reason, it has been postulated that parasites that are transmitted exclusively vertically will either evolve towards mutualism or disappear from the host population (Bull, Molineux \& Rice, 1991; Frank, 1991, 1996). Further, vertical transmission is usually uniparental, and the nontransmitting sex effectively becomes a dead-end for vertical transmission. In microsporidia, it has been suggested that vertical transmission evolved several times (Smith \& Dunn, 1991; Dunn \& Smith, 2001), being considered an important adaptation to fluctuating environments in this group of parasites (Lucarotti \& Andreadis, 1995). Most vertically transmitted microsporidia, however, also transmit horizontally (Becnel \& Andreadis, 1999; Dunn \& Smith, 2001). Mixed routes of transmission could provide a way of overcoming the constraints imposed by either route alone (Agnew et al. 2002).

The microsporidium Octosporea bayeri Jírovec 1936 was first described as a parasite of Daphnia magna in the Lednice Biological Station, Czech Republic. Jírovec (Jírovec, 1936) described O. bayeri infecting the fat cells of D. magna, which suffered hypertrophy due to spore proliferation. O. bayeri was later found in D. magna populations of the rock-pools of the Tvärminne Archipelago in Southern Finland (Green, 1957) and in Hampton Court, South of London (Green, 1974). The Tvärminne D. magna populations are inherently unstable due to the frequent drying up of the rock pools, followed by sudden invasion of brackish or rain water (Ebert, Hottinger \& Pajunen, 2001; Haag et al. 2002). The prevalence of $O$. bayeri in this metapopulation, however, is high, with $45 \%$ of the populations containing infected hosts, and an average prevalence close to $50 \%$ within those populations (Ebert et al. 2001). To achieve high prevalences in such an unstable environment $O$. bayeri requires strategies to survive frequent phases of low host density and local host extinctions. This study investigates the infection mechanisms of $O$. bayeri, and aims at elucidating its transmission routes in the context of some of the environmental constraints this parasite faces in its natural Daphnia metapopulation.

\section{MATERIALS AND METHODS}

\section{System}

Isolates of Octosporea bayeri were produced by collecting single-infected female Daphnia magna from rock-pool populations in the Tvärminne Archipelago, Southern Finland, and allowing them to reproduce clonally to produce iso-female host lines. The infection can be maintained in the laboratory in D. magna populations obtained from such iso-female host lines. Spores can be collected from dying or dead individuals to infect new host clones through horizontal transmission. The O. bayeri isolates were cultured in their original host clones, or in novel host clones. All cultures were kept in $100-400 \mathrm{ml}$ of artificial medium (Klüttgen et al. 1994; modified after Ebert, Zschokke-Rohringer \& Carius (1998)) at $20{ }^{\circ} \mathrm{C}$ in a dark: light cycle of $8: 16 \mathrm{~h}$, and fed regularly with the green algae Scenedesmus sp. All experiments were done under these conditions, unless stated otherwise. Table 1 summarizes the experiments performed and their main results. 


\section{Pathology of infection and spore morphology}

To observe the tissues affected by $O$. bayeri, infected female and male D. magna from stock cultures were isolated and anaesthetized in a dilution of carbonized water for up to $2 \mathrm{~min}$. The animals were then placed in a drop of medium on glass slides and observed using a phase-contrast microscope at 40 to $100 \times$ magnification. Pictures were taken with a FireWire Video Camera. To observe the spores, whole $D$. magna of various ages were placed on glass slides with a drop of medium. A cover-slip was pressed upon the Daphnia with a rotating motion so as to spread the tissues evenly. These wet-mount preparations were analysed with phase-contrast microscopy, magnification ranging between 300 and $600 \times$. Pictures of spores were taken from such wet-mount preparations, with a FireWire Video Camera, and with a Nikon E990. Spores were measured directly from digital images using the public domain software Object-Image2.10 (http://simon.bio.uva.nl/).

\section{Horizontal transmission}

Experiment 1. To determine if the rate of horizontal infection depends on the concentration of spores in the medium, a dose experiment was performed. Uninfected D. magna females of 3 days of age were singly placed in $20 \mathrm{ml}$ of medium containing different amounts of $O$. bayeri spores $(0,10,100,1000$, 10000 , and 100000$), 12$ individuals per spore-dose. After 4 days $80 \mathrm{ml}$ of medium were added to each jar, and the Daphnia dissected for observation at day 18 post-infection. The infective status was determined by the presence of spores (at $600 \times$ ).

Experiment 2. To determine when horizontal transmission occurred, a set of controlled exposures of uninfected D. magna to hosts infected with 3 isolates of $O$. bayeri were performed. Juvenile, infected Daphnia from mass cultures (15 female and 15 male donors per isolate) were transferred individually into $100 \mathrm{ml}$ of medium, and served as spore donors. Uninfected female Daphnia of 3 days of age served as recipients, and were put in contact with the spore donors at 4 consecutive times. On the day of transfer, 2 recipients were added to each jar (T1). After 6 days, the recipients were transferred to fresh medium. The procedure was repeated with a second batch of recipients, using the same spore donors (now 6 days older, T2). Uninfected recipients were further added whenever a spore donor died (T3), and 6 days after the spore donors death (T4). For the last exposure (T4), the body of the dead spore donors was homogenized and the spore solution was added to $100 \mathrm{ml}$ of medium. All recipients were kept in contact with the spore donor (T1-T3) or in the medium with spores (T4) for 6 days. All exposed Daphnia were allowed to reproduce clonally, and the presence of spores was assessed in both mothers and offspring about 1 month after exposure. To test for the effect of parasite isolate, donor sex, and time of exposure on the frequency of infection, the data were fitted with a Logistic Regression (Kleinbaum et al. 1998).

\section{Environmental persistence of spores}

Experiment 3. Spores in solution. Medium containing a variable amount of dead Daphnia infected with 3 isolates of $O$. bayer $i$ was maintained at $20{ }^{\circ} \mathrm{C}$ in $100 \mathrm{ml}$ jars, without adding fresh medium. A total of 22 jars were used $(8,7$, and 7 for each isolate respectively). After 24 days 5 uninfected 4 to 10-day-old female Daphnia were added to the medium. The females were transferred individually to fresh medium when they matured, and the presence of spores was determined in the survivors after they had produced their second clutch of eggs. The effect of the parasite isolate on the frequency of infection was tested with the Likelihood Ratio (Kleinbaum et al. 1998).

Experiment 4. Desiccation of spores. Spore resistance to desiccation was tested by determining the infectivity of spores after a period of desiccation. Populations of D. magna infected with combinations of $O$. bayeri isolates were kept in $400 \mathrm{ml}$ jars for 4 months. The sediment accumulated during this period was spread over filter paper and allowed to dry at $20^{\circ} \mathrm{C}$ with shaded artificial light during 5 weeks. The sediment was then exposed to continuous artificial light at $32{ }^{\circ} \mathrm{C}$ during 3 days and resuspended in $100 \mathrm{ml}$ of medium. Ten uninfected Daphnia (ages 3 to 15 days) were added to each of these suspensions. The presence of spores in all individuals and their offspring was determined in wet mounts after 4 weeks. A total of 107 replicates (sediment from independent populations), infected with combinations of 7 different parasite isolates (2-4 replicates per isolate combination) were used.

\section{Vertical transmission}

Experiment 5. Transmission to parthenogenetic offspring. Mature female Daphnia infected with 1 isolate of $O$. bayeri were kept individually in $100 \mathrm{ml}$ of medium and checked 4 times per day to assess their reproductive state. Daphnia with full ovaries but empty brood chambers were observed every $10 \mathrm{~min}$ until new eggs were released into the brood chamber. The eggs were then carefully flushed out from the brood chamber of the living mother using a Pasteur pipette, and transferred individually into $2.5 \mathrm{ml}$ of fresh medium. After eclosion, the Daphnia were transferred to $100 \mathrm{ml}$ of medium, fed regularly, and analysed for the presence of spores when they were 1 month old. 
Experiment 6. Transmission to sexual offspring. Sexual eggs (= resting eggs, ephippia) from 126 laboratory populations of Daphnia infected with combinations of 7 isolates of $O$. bayeri were accumulated in the cultures' sediment during 4 months as described in Exp. 3. Briefly, the ephippia were dried along with the sediment from the jars at $20^{\circ} \mathrm{C}$ during 5 weeks, then exposed to continuous artificial light at $32{ }^{\circ} \mathrm{C}$ during 3 days. The ephippia were carefully washed under running water for $5 \mathrm{~min}$ to remove sediment and spores that might stick to their surface, and were then placed per population in $50 \mathrm{ml}$ of medium at $20^{\circ} \mathrm{C}$ until hatching. The hatched Daphnia of each population were kept in the same jar until they reproduced, thus producing a new population that was allowed to grow up to the second generation. During the growth of the populations we allowed horizontal transmission, thus increasing the possibilities of detecting the infection even if only 1 of the hatched Daphnia had carried the infection through the ephippial phase. The presence of spores was determined in 10 randomly chosen adults (both hatched Daphnia and their offspring) from each of these new populations. Additionally, groups of 5 ephippia from 4 populations were immersed in diluted commercial bleach for 1, 2, or $5 \mathrm{~min}$, to ensure no viable spores remained on the surface. These groups of 'bleached' ephippia were then grown into populations as above.

To further determine whether ephippia acquire the infection vertically or through spores attached in the surface, embryos were removed from 20 ephippia from infected populations, and washed carefully in medium. The embryos were then analysed with a PCR probe to detect the presence of microsporidian SSUrDNA as described by Canning et al. (2002). Briefly, DNA was extracted with the DNeasy Tissue Kit (Qiagen) and PCR was carried out using the microsporidian primers Pmp1 (5'-CACCAGGTT GATTCTGCCTGAC-3') and HG4r (5'-TGGTC CGTGTTTCAAGACGGG-3') (Zhu et al. 1993; Gatehouse \& Malone, 1998).

\section{RESULTS}

\section{Pathology of infection and spore morphology}

As described by Jírovec (1936), D. magna infected with $O$. bayeri appear opaque, the inner tissues filled with a pale mass of spores (Fig. 1). In the Daphnia studied here, the infection was found not only in the fat cells (Jírovec, 1936; Green, 1957, 1974), but also in the ovary. This can be observed in whole animals, as the mass of spores invades the ovaries, making them opaque (arrow in Fig. 1A). In advanced stages of infection, the mass of spores spreads through the entire body cavity (Fig. 1B).

Spores varied in size, shape, and refringency, and could be classified into 3 morphological types (Fig. 2). One kind of spore, is oval in shape and
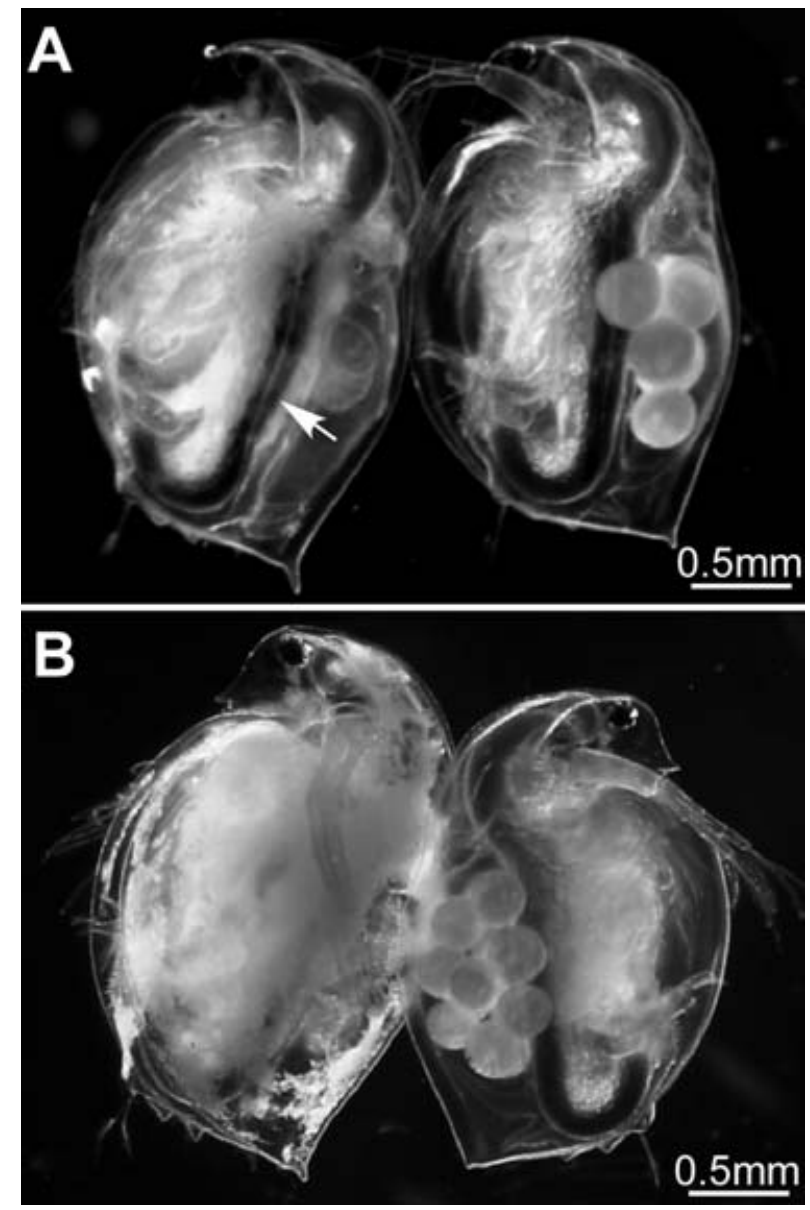

Fig. 1. Morphology of infection with Octosporea bayeri. (A) Mature infected (left) and uninfected (right) Daphnia magna females. The spores of O. bayeri accumulate in the fat cells and in the ovary (arrow). (B) An advanced infection (left) showing the spread of spores throughout the body cavity of a female D. magna. Uninfected female (right) shown for comparison.

refringent under the phase-contrast microscope (Fig. 2A), and between 4.9 and $5.6 \mu \mathrm{m}$ long and $2 \cdot 2$ and $2 \cdot 3 \mu \mathrm{m}$ wide (321 spores measured in 34 hosts, 2 to 15 spores/host, 8 parasite isolates). Empty shells of germinated spores of this type can be found in wet-mount preparations (arrow in Fig. 2A), allowing the measurement of the spore wall, which was $0 \cdot 49-0.53 \mu \mathrm{m}$ thick (23 spores measured). The thick spore wall prevents the visualization of the sporoplasm using light microscopy. Germination is enhanced in wet-mount preparations when pressure is increased (Fig. 3A). The second spore type shown in Fig. $2 \mathrm{~B}$ varies between 4 and $5 \cdot 2 \mu \mathrm{m}$ in length and $1 \cdot 6$ and $2 \cdot 1 \mu \mathrm{m}$ in breadth (286 spores measured in 36 hosts, 1 to 15 spores/host, 8 parasite isolates). It has a pear-like shape, and the sporoplasm can be seen in phase-contrast. Its reduced refringency suggests a thinner spore-wall. Germination occurs frequently in wet-mount preparations, suggesting that this spore type actually corresponds to mature spores and not to early stages of the spore type described above (Fig. 3B). A third spore type (Fig. 2C), less 

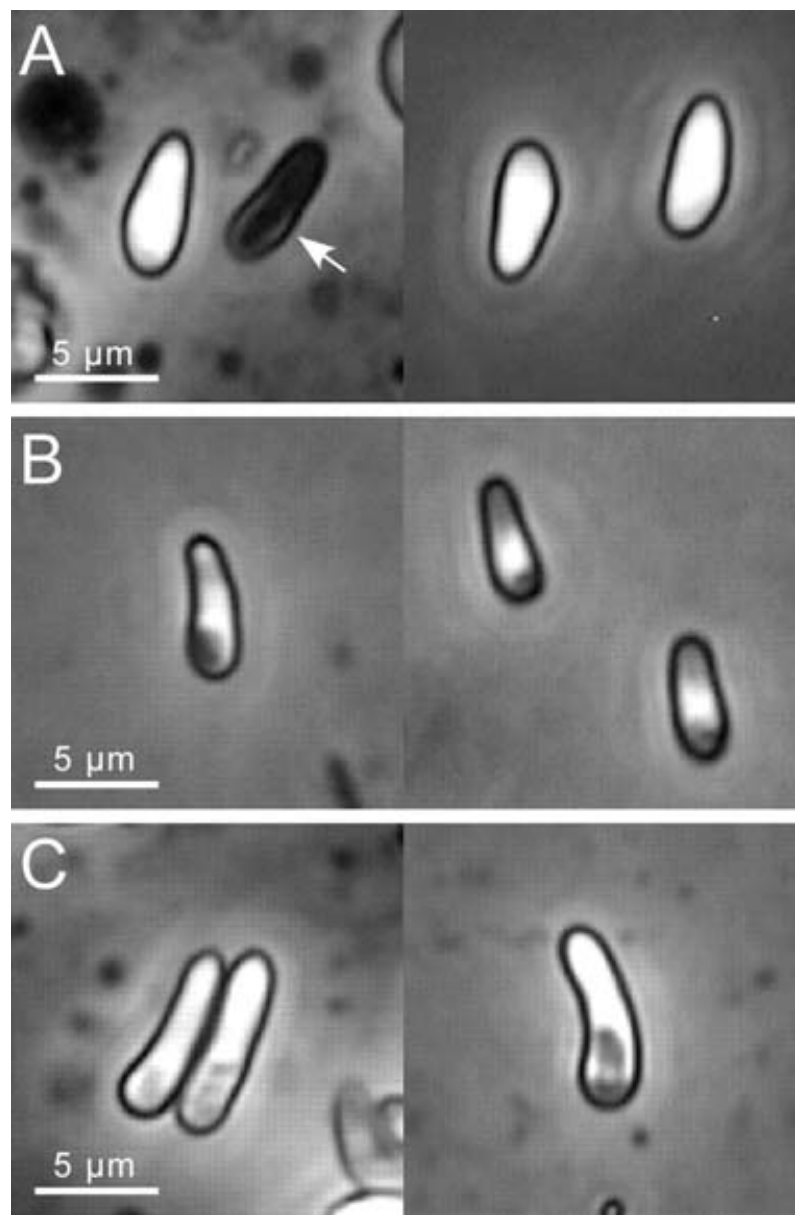

Fig. 2. Spore types of Octosporea bayeri observed with phase-contrast microscopy. (A) Refringent spores. The arrow indicates an empty spore shell, probably after germination. (B) Non-refringent spores. (C) Spores of intermediate refringency.

frequently observed than the previous spore types, is of intermediate refringency (spore wall 0.35$0.45 \mu \mathrm{m}, 29$ spores measured) and elongated in shape, straight or slightly curved. This kind of spore varies from 6.8 to $12 \mu \mathrm{m}$ in length, and between 1.6 and $2 \cdot 1 \mu \mathrm{m}$ in breadth (163 spores measured in 22 hosts, 1 to 12 spores/host, 6 parasite isolates). Germination of this spore type can also be observed in wet-mount preparations (Fig. 3C), the polar tube being up to $80 \mu \mathrm{m}$ long. Although the different spore types observed in $O$. bayeri possibly correspond to typical microsporidian spore types, ultimate tests of the role of each spore type would require ultrastructural studies, type-specific separation and infection, assays of environmental persistence, and intra-host germination.

\section{Horizontal transmission}

Unsurprisingly, horizontal transmission of O. bayeri depends on the concentration of spores present in the medium, as shown by the results of Exp. 1. Figure 4 shows the infectivity (percentage of infected
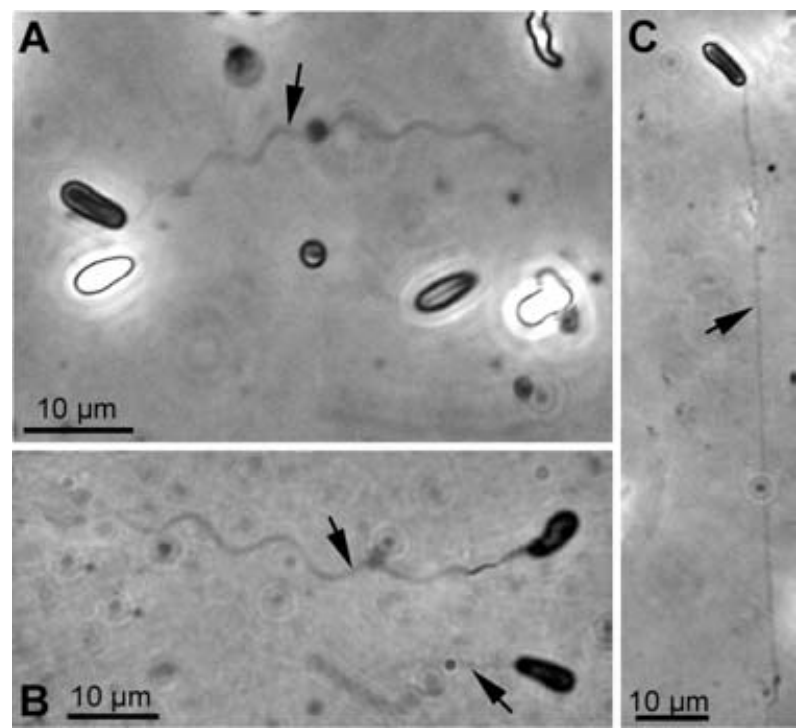

Fig. 3. Germinated Octosporea bayeri spores in wet mount preparations. (A) Germinated type-A spore. (B) Germinated type-B spores. (C) Germinated type-C spores of intermediate refringency. Arrows indicate polar tubes.

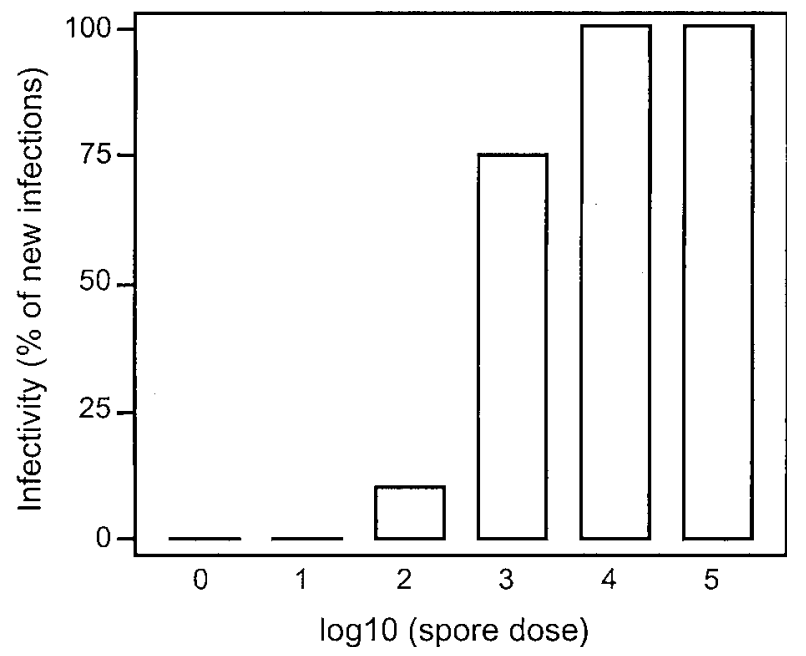

Fig. 4. Percentage of Daphnia magna infected when exposed to different spore-doses of Octosporea bayeri. The columns represent the infectivity for each sporedose, calculated as the $\log _{10}$ of the total number of spores added to $20 \mathrm{ml}$ of medium.

individuals) for each spore-dose $\left(\log _{10}\right.$ of number of spores in $20 \mathrm{ml}$ ). A minimum of 100 spores in $20 \mathrm{ml}$ was required for any infection to occur, and 10000 spores were enough to acquire $100 \%$ infection.

The results of Exp. 2 suggest that horizontal transmission of O. bayeri occurs only after host death. Uninfected Daphnia exposed to living infected hosts neither developed spores, nor produced infected offspring (Fig. 5 ; Exp. 2). Only recipients exposed to the spore donors within the 6 days after their death developed infections. The proportion of infections increased significantly after the dead hosts were 


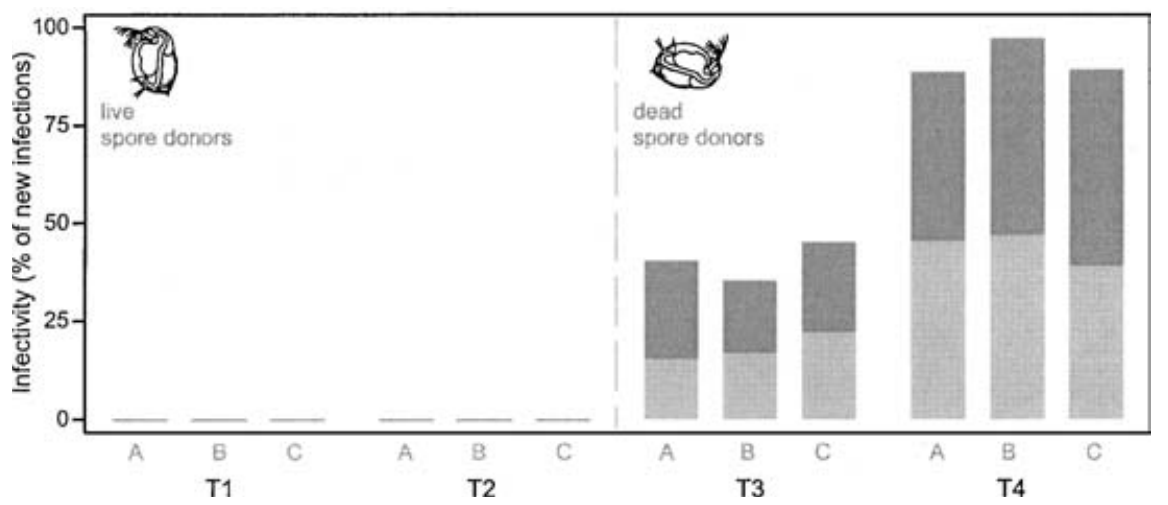

Fig. 5. Percentage of infections with three isolates of Octosporea bayeri obtained when exposing young uninfected Daphnia magna to spore donors at 4 different times (T) of their lives (see text for details). T1 and T2 are separated by 6 days, and the spore donors were alive. At T3 the spore donors had just died, and at T4 the host was crushed after been dead for at least 6 days. A, B and $\mathrm{C}$ represent the 3 parasite isolates, and different greys represent the sex of the spore donor (dark for female and light for male).

crushed (Likelihood Ratio Chi-Square $=45 \cdot 3$, $P<0 \cdot 0001)$ with no influence of parasite isolate (Likelihood Ratio Chi-Square $=0 \cdot 15, P=0 \cdot 92$ ) or spore donor sex (Likelihood Ratio Chi-Square $=$ $1 \cdot 33, P=0 \cdot 25)$.

\section{Environmental persistence of spores}

Spores remained infective when kept in aqueous solution at $20{ }^{\circ} \mathrm{C}$ for 24 days, as shown by the results of Exp. 3. Overall, 36.4\% of jars containing medium with spores produced infections. In those jars, all surviving Daphnia were infected. The parasite isolate did not have a significant effect on infectivity (Likelihood Ratio Chi-Square $=0.32, \quad P=0.85$ ). Further, uninfected female Daphnia that were exposed to spores desiccated for 5 weeks and resuspended in medium acquired the infection in $54 \%$ of the cases (107 replicates across all parasite isolates, Exp. 4).

\section{Vertical transmission}

In Exp. 5, a total of 79 parthenogenetic eggs removed from the brood chamber of 27 infected Daphnia eclosed and survived until spore analysis. All individuals contained spores, suggesting that vertical transmission to parthenogenetic offspring is near to $100 \%$ efficient, and that the infection occurs before or during egg release into the brood chamber of the mother.

Ninety-eight of the 126 replicates (78\% hatching success) of Exp. 6 produced a new population of Daphnia. Among those, only $84 \%$ of populations resulted in infection with $O$. bayeri. The ephippia that were immersed in bleach had $67 \%$ hatching success, and all developed into infected females. The presence of the parasite in the embryos was further confirmed in 18 of the 20 ephippia analysed with PCR. Therefore infection to ephippia seems to occur vertically and not through spores in the sediment but, in contrast to the vertical transmission to parthenogenetic offspring, vertical transmission to sexual eggs is less than $100 \%$ efficient.

\section{Parasite life-cycle}

Given the observations on transmission of O. bayeri, a life-cycle of the parasite in its host can be proposed (Fig. 6). Horizontal transmission (lower part of Fig. 6) occurs when infected female or male hosts die and spores are released to the environment. Environmental spores survive outside the host for at least short periods of time in different conditions, increasing the chances of infecting new hosts even after brief periods of local extinction of the host. An infected female can also transmit the parasite to its parthenogenetic offspring through direct vertical transmission. This transmission route is maintained during the parthenogenetic cycle of D. magna (upper part of Fig. 6). Vertical transmission also occurs in the sexual cycle, likely through the mother. Finally, ephippia may serve as a vehicle for parasite dormancy and dispersal, with a new cycle of vertical and/or horizontal transmission starting after hatching.

\section{ISCUSSION}

\section{Transmission and persistence}

Horizontal transmission of $O$. bayeri occurred only after host death. This is characteristic of microsporidia infecting non-epithelial tissues, where the release of spores requires some degree of tissue destruction before the spores can be released (Becnel $\&$ Andreadis, 1999). By the time of death, most infected hosts are filled with spores, as the parasite grows rapidly inside the host, as long as the host is still alive (Vizoso \& Ebert, 2004). As suggested by the increase of infectivity when hosts were crushed, the rupture of spore-containing tissues in D. magna does 


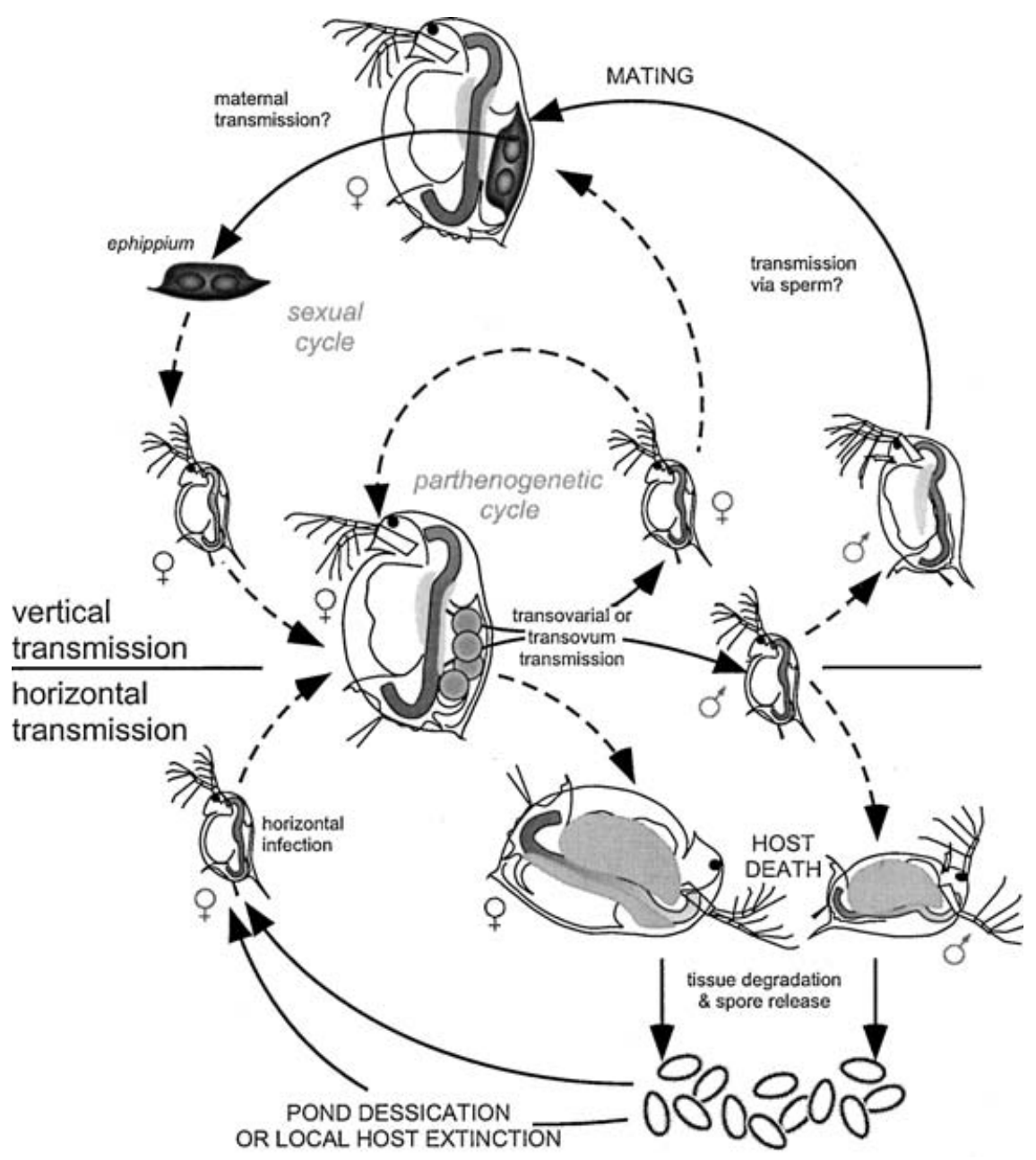

Fig. 6. Suggested life-cycle of Octosporea bayeri infecting Daphnia magna. Grey stippled lines indicate within-host parasite proliferation. See text for details.

not occur immediately after host death. In nature, this process may be enhanced by bacterial and detritivore activity. Moreover, D. magna often browses over the sediment, stirring up particles that are then ingested by filter feeding (Ebert, 1995), and may therefore ingest spores present in decaying infected D. magna.

The survival of spores outside the host, both dry and in wet conditions, suggests that spores may be able to persist in the field in the absence of susceptible hosts. This may in turn enhance the permanence and prevalence of O. bayeri in the D. magna metapopulations. First, spores remained viable in aqueous solution for at least 1 host generation. The chances of new infections may thus increase as susceptible hosts might encounter viable spores even 24 days after the spore donor died. Even if an infected population would become extinct, susceptible $D$. magna that re-colonize the pond may thus acquire the infection, maintaining the parasite in the pond. Second, spores accumulated in the sediment remained viable after 5 weeks of desiccation. In the natural habitat, ponds dry frequently during midsummer. In this situation, producing spores resistant to desiccation may allow O. bayeri to infect hosts that either hatch from ephippia or invade the rock pool once rainwater has refilled it. Resistance of spores to desiccation and light was thought to be absent in aquatic microsporidia (Becnel \& Andreadis, 1999), and may be a particular adaptation of $O$. bayeri to the highly unstable environmental conditions found in the rock pools of the Finnish metapopulation. It is also possible that the presence of sediment (in our experiment as well as often in the field) enhances spore survival, by offering some protection from light and a source of moisture.

$O$. bayeri showed a highly efficient vertical transmission to parthenogenetic offspring. In field situations of low host density and little or no competition with uninfected hosts, vertical transmission to parthenogenetic offspring may allow O. bayeri to remain in the host population. D. magna hatching from ephippia were also infected. In the field, this route of infection may bring two advantages to the parasite. First, it could provide a way of surviving the extreme conditions usually present during host diapause. Infecting ephippia would be a safe way of ensuring that (at least some) infected hosts will continue 
the cycle after diapause is over. Relying solely on the persistence of spores may result in local parasite extinction, as spores might die or be removed by wind or water. The thick, melanized coat of the ephippia may also protect the parasite from the environmental conditions. The second advantage of parasitizing ephippia is dispersal. In systems of rock pools, ephippia can be passively dispersed by water, birds or wind (Ranta, 1979). Parasitized ephippial eggs may thus serve as vehicles for parasite dispersal, potentially spreading the infection throughout the rock pool system. As the D. magna metapopulation in which $O$. bayeri is found is highly dynamic with $20 \%$ extinctions per pool per year (Pajunen, 1986) effective dispersal is essential for persistence in the host population.

Although our experiments suggest that the persistence of $O$. bayeri might be high, Ebert et al. (2001) showed that pools recolonized by Daphnia are usually free of parasites. Other ecological factors that may be limiting parasite persistence in this system are over-wintering (e.g. resistance to low temperatures), immigration of resistant clones, and removal of spores and ephippia by water or wind.

\section{Mechanisms of vertical transmission}

The results of Exp. 3 (transmission to parthenogenetic offspring) suggest that vertical transmission occurs prior to egg release into the brood chamber. Although the infection could still occur in the brood chamber during the first $10 \mathrm{~min}$, the high transmission efficiency and the absence of any horizontal transmission while the hosts are alive make this possibility unlikely. The mass of spores observed in the ovaries further suggests that transmission may be transovarial. However, eggs could also be infected in the passage from the ovary to the brood chamber (transovum infection, Canning, 1982). Conclusive evidence should include ultrastructural analyses of infected D. magna ovaries.

The results of Exp. 4 suggest that sexual eggs are also infected vertically. Infection via spores attached to the ephippia is unlikely. First, embryos extracted from the ephippia contained copies of the parasite DNA. Second, the percentage of infections in ephippia exposed to the sediment was considerably lower than that of the ephippia coming from infected populations (Exp. 4). Vertical transmission to sexual offspring through males also seems unlikely, as no male-to-female infection was observed in Exp. 1, and no cases of transmission via sperm are known (Becnel $\&$ Andreadis, 1999). The exposed females, however, might have been too young for mating to occur. Therefore, experiments that explicitly assess the possibility of paternal transmission are required.

We cannot compare the efficiencies of the parthenogenetic and the sexual transmission with our experimental set up. However, it seems safe to conclude that whereas parthenogenetic transmission in these isolates rarely fails (which has been repeatedly corroborated during the keeping of the cultures), the transmission to sexual offspring is not as efficient, and offers a way of purging the parasite from an infected Daphnia clone. Production of uninfected sexual offspring by infected Daphnia may be due to differences in the process of parasite transmission to the egg or embryo, a hypothesis that requires thorough examination (e.g. through ultrastructural analyses). It is also possible that the embryo is able to survive the extreme conditions (high temperatures, drought) whereas the parasite is not. Another possibility, more exciting but difficult to test, is that sexual offspring may have acquired resistance to the maternal parasite strain through recombination. In both cases, if transmission to sexual offspring is not perfect, sexual reproduction could allow infected populations to reduce parasite prevalence.

In conclusion, our results suggest that both vertical and horizontal transmission are required for the persistence of $O$. bayeri under the harsh conditions the host metapopulation is exposed to, and that the advantages of each route may compensate the disadvantages of the other. Vertical transmission is probably crucial during periods of low host density and may play an important role in parasite dispersal. On the other hand, horizontal transmission, through the release of large quantities of spores that may boost the parasite's prevalence in each pond, is necessary to counteract the negative effects of between-host competition that would arise due to vertical transmission.

Some of the pictures were taken in the lab of Nico Michiels with the help of Lukas Schärer. Christoph Haag and Jürgen Hottinger helped with the collection of some of the samples. Some of the parasite isolates were maintained in the laboratory by J. Hottinger, Patrick Mucklow, and Marc Zbinden. J. Hottinger and Lusia Sygnarski provided invaluable laboratory assistance. Marc Capaul performed the dose-experiment. D. B. V. and S. L. further profited from discussions with D. Refardt and M. Zbinden. DBV received financial support from CONICIT (Venezuela) and the Roche Research Foundation (Switzerland, grant 2002111). S. L. acknowledges funding by the German Research Foundation (DFG, grant LA 1400/1-1). The laboratory material was financed by the Swiss National Foundation.

\section{REFERENCES}

AGNEW, P., BECNEL, J. J., EBERT, D. \& MICHALAKIS, Y. (2002).

Symbiosis of microsporidia and insects. In Insect

Symbiosis (ed. Bourtzis, K.), pp. 145-163. CRC Press LLC, Florida.

ANDERSON, R. M. \& MAY, R. M. (1978). Regulation and stability of host-parasite population interactions.1. Regulatory processes. Fournal of Animal Ecology 47, 219-247.

ANDERSON, R. M. \& MAY, R. M. (1981). The population dynamics of micro-parasites and their invertebrate 
hosts. Philosophical Transactions of the Royal Society of London, Series B 291, 451-524.

ANDERSON, R. M. \& MAY, R. M. (1986). The invasion, persistence and spread of infectious-diseases within animal and plant-communities. Philosophical Transactions of the Royal Society of London, Series B 314, 533-570.

BECNEL, J. J. \& ANDREADIS, T. G. (1999). Microsporidia in insects. In The Microsporidia and Microsporidiosis (ed. Wittner, M. \& Weiss, L. M.), pp. 447-501. American Society for Microbiology, Washington, D.C. BIGLIARDI, E. \& SACCHI, L. (2001). Cell biology and invasion of the Microsporidia. Microbes and Infection 3, 373-379.

BONHOEFFER, S., LENSKI, R. E. \& EBERT, D. (1996). The curse of the pharaoh: The evolution of virulence in pathogens with long living propagules. Proceedings of the Royal Society of London, Series B 263, 715-721.

BRooks, w. M. (1988). Entomogenous protozoa. In $C R C$ Handbook of Natural Pesticides. Microbial Insecticides, Part A : Entomogenous Protozoa and Fungi (ed. Ignoffo, C. M.), pp. 1-150. CRC Press, Boca Raton, Florida.

BULl, J. J., MOLINEUX, I. J. \& RICE, W. R. (1991). Selection of benevolence in a host-parasite system. Evolution 45, 875-882.

CANNING, E. (1982). An evaluation of protozoal characteristics in relation to biological control of pests. Parasitology 84, 119-149.

CANNing, E. U., REFARDT, D., VOSSBRINCK, C. R., OKAMURA, B. \& CURRY, A. (2002). New diplokaryotic microsporidia (Phylum Microsporidia) from freshwater bryozoans (Bryozoa, Phylactolaemata). European Fournal of Protistology 38, 247-265.

DUNN, A. M. \& SMITH, J. E. (2001). Microsporidian life cycles and diversity: the relationship between virulence and transmission. Microbes and Infection 3, 381-388.

EBERT, D. (1995). The ecological interactions between a microsporidian parasite and its host Daphnia magna. Fournal of Animal Ecology 64, 361-369.

EBERT, D., HOTTINGER, J. W. \& PAJUnEN, v. I. (2001). Temporal and spatial dynamics of parasite richness in a Daphnia metapopulation. Ecology 82, 3417-3434.

EBERT, D., ZSCHOKKE-ROHRINGER, C. D. \& CARIUS, H. J. (1998). Within- and between-population variation for resistance of Daphnia magna to the bacterial endoparasite Pasteuria ramosa. Proceedings of the Royal Society of London, Series B 265, 2127-2134.

FRANK, S. A. (1991). Ecological and genetic models of host-pathogen coevolution. Heredity 67, 73-83.

FRANK, S. A. (1996). Models of parasite virulence. Quarterly Review of Biology 71, 37-78.

Gatehouse, H. S. \& MALONE, L. A. (1998). The ribosomal RNA gene region of Nosema apis (Microspora): DNA sequence for small and large subunit rRNA genes and evidence of a large tandem repeat unit size. Fournal of Invertebrate Pathology 71, 97-105.

Green, J. (1957). Parasites and epibionts of Cladocera in rock pools of Tvärminne archipelago. Archivum Societatis Zoologicae Botanicae Fennicae "Vanamo" 12, 5-12.
GREEN, J. (1974). Parasites and epibionts of Cladocera. Transactions of the Zoological Society of London 32, 417-515.

HAAG, C. R., HOTTINGER, J. W., RIEK, M. \& EBERT, D. (2002). Strong inbreeding depression in a Daphnia metapopulation. Evolution 56, 518-526.

Jírovec, o. (1936). Über einige in Daphnia magna parasitierende Mikrosporidien. Zoologischer Anzeiger 116, 136-142.

KLeinbaUm, D. G., KUPPER, L. L., MUller, K. E. \& NiZAM, A. (1998). Applied regression Analysis and Other Multivariate Methods. Duxbury Press,

Pacific Grove, CA.

KLÜTTGEN, B., DÜLMER, U., ENGELS, M. \& RATTE, H. T. (1994). ADaM, an artificial freshwater for the culture of zooplankton. Water Research 28, 743-746.

LUCAROTTI, C. J. \& ANDREADIS, T. G. (1995). Reproductive strategies and adaptations for survival among obligatory microsporidian and fungal parasites of mosquitoes : a comparative analysis of Amblyospora and Coelomyces. Fournal of the American Mosquito Control Association 11, 111-121.

MADDOX, J. v. (2002). Environmental persistence of Microsporidia. In Factors Affecting the Survival of Entomopathogens (ed. Baur, M. E. \& Fuxa, J. R.). Southern Cooperative Series Bulletin 400. Available at: http://www.agctr.lsu.edu/s265/default.htm

ONSTAD, D. W., MADDOX, J. V., COX, D. J. \& KORNKVEN, E. A. (1990). Spatial and temporal dynamics of animals and the host-density threshold in epizootiology. Fournal of Invertebrate Pathology 55, 76-84.

Pajunen, v. I. (1986). Distributional dynamics of Daphnia species in a rock-pool environment. Annales Zoologici Fennici 23, 131-140.

RANTA, E. (1979). Niche of Daphnia species in rock pools. Archiv für Hydrobiologie 87, 205-223.

REGOES, R. R., HOTTINGER, J. W., SYGNARSKI, L. \& EBERT, D. (2003). The infection rate of Daphnia magna by Pasteuria ramosa conforms with the mass-action principle.

Epidemiology and Infection 131, 957-966.

SaUvage, F., Langlais, M., YocCOZ, N. G. \& PONTIER, D. (2003). Modelling hantavirus in fluctuating populations of bank voles: the role of indirect transmission on virus persistence. Fournal of Animal Ecology 72, 1-13.

SMiTh, J. E. \& DUNN, A. M. (1991). Transovarial transmission. Parasitology Today 7, 146-148.

van baalen, M. (2000). Parent-to-offspring infection and the struggle for transmission. In Evolutionary Biology of Host-Parasite Relationships: Theory Meets Reality (ed. Poulin, R., Moran, S. \& Skorping, A.), pp. 97-116. Elsevier, Amsterdam.

VIzoso, D. B. \& EBERT, D. (2004). Within-host dynamics of a microsporidium with horizontal and vertical transmission: Octosporea bayeri in Daphnia magna. Parasitology 128, 31-38.

ZHU, X., WitTNER, M., TANOWITZ, H. B., CALI, A. \& Weiss, L. M. (1993). Nucleotide sequence of the small ribosomal RNA of Encephalitozoon cuniculi. Nucleic Acids Research 21, 1315. 